Article

\title{
Some Features of Rank One Real Solvable Cohomologically Rigid Lie Algebras with a Nilradical Contracting onto the Model Filiform Lie Algebra $Q_{n}$
}

\author{
Rutwig Campoamor-Stursberg $1,2, * \mathbb{1}$ and Francisco Oviaño García ${ }^{2}$ \\ 1 Instituto de Matemática Interdisciplinar, Universidad Complutense de Madrid, Plaza de Ciencias 3, \\ E-28040 Madrid, Spain \\ 2 Dpto de Geometría y Topología, Fac. CC. Matemáticas, Universidad Complutense de Madrid, \\ Plaza de Ciencias 3, E-28040 Madrid, Spain; foviano@ucm.es \\ * Correspondence: rutwig@ucm.es
}

Received: 25 November 2018; Accepted: 14 January 2019; Published: 16 January 2019

\begin{abstract}
The generic structure and some peculiarities of real rank one solvable Lie algebras possessing a maximal torus of derivations with the eigenvalue spectrum $\operatorname{spec}(\mathfrak{t})=$ $(1, k, k+1, \cdots, n+k-3, n+2 k-3)$ for $k \geq 2$ are analyzed, with special emphasis on the resulting Lie algebras for which the second Chevalley cohomology space vanishes. From the detailed inspection of the values $k \leq 5$, some series of cohomologically rigid algebras for arbitrary values of $k$ are determined.
\end{abstract}

Keywords: real Lie algebra; solvable; rigidity; cohomology; contraction

\section{Introduction}

In a wide sense, the notion of rigidity of Lie algebras can be understood as the stability of its defining structure tensor with respect to some action of a group or a deformation, implying that whatever the alteration of the structure tensor defined by geometric or algebraic means, the resulting Lie algebra is isomorphic to the starting one. Although originally developed in the context of smooth manifolds and functional spaces, the rigidity concept has turned out to be very important in the Lie algebraic frame, not only for classification purposes, but also for the geometric study of the variety determined by the structure tensor of Lie algebras in a given dimension [1], its irreducible components and various limiting processes on the variety [2]. The development of Lie algebra cohomology [3], as well as its implications in the theory of deformations and contractions $[2,4,5]$ motivated the search for rigidity criteria beyond the well-known case of semisimple algebras, which follows as a consequence of the classical Whitehead lemmata [6,7]. The rigidity of Lie algebras has been analyzed from various perspectives, ranging from the geometrical and topological point of view in terms of the so-called Jacobi schemes [8] to the pure algebraic approach, based on the Chevalley cohomology of Lie algebras [3] or the root theory of solvable Lie algebras relying on the study of the eigenvalue spectrum of generators of maximal tori of derivations of nilpotent algebras. This generalizes naturally the approach of roots systems of semisimple Lie algebras [9,10]. In this context, large classes of rigid Lie algebras in the cohomological sense have been classified (see, e.g., [11-18] and references therein), albeit it is well known that the rigidity notion goes far beyond the cohomological criteria [19,20], even allowing the existence of rigid Lie algebras that can be purely nonrational and nonreal [21].

In this work, we consider solvable Lie algebras $\mathfrak{r}$ of rank one possessing a maximal torus of derivations $\mathfrak{t}$, the spectrum of which is given by the eigenvalues $\operatorname{spec}(\mathfrak{t})=$ $(1, k, k+1, \cdots, n+k-3, n+2 k-3)$ on its $n$-dimensional nilradical $\mathfrak{n}$. These nilradicals are known to contract onto the so-called $Q_{n}$-model filiform algebra, a nilpotent Lie algebra of maximal nilpotence 
index with the additional property of satisfying the condition $[[\mathfrak{n}, \mathfrak{n}],[\mathfrak{n}, \mathfrak{n}]] \neq 0$ [22]. Analyzing in detail these nilradicals $\mathfrak{n}$ for low values of $k$, we concentrate on the resulting solvable Lie algebras that are rigid in the cohomological sense. Based on certain patterns observed for these low values, we extrapolate the result to obtain various infinite series of rigid Lie algebras.

This work complements and extends the existing analysis of rank one solvable rigid Lie algebras, following the same motivations and techniques used in the works $[9,13,15,23,24]$, where the eigenvalue $\operatorname{spectra} \operatorname{spec}(\mathfrak{t})=(1,2,3, \cdots, n), \operatorname{spec}(\mathfrak{t})=(1,3,4, \cdots, n+1)$ and $\operatorname{spec}(\mathfrak{t})=(1,4,5, \cdots, n+2)$ are studied in detail.

Unless otherwise stated, any Lie algebra considered in this work is finite-dimensional and defined over the real field $\mathbb{K}=\mathbb{R}$.

\subsection{Solvable Real (Rigid) Lie Algebras}

Let $\mathfrak{g}$ be a Lie algebra and $\operatorname{Der}(\mathfrak{g})$ denote the Lie algebra formed by its derivations, i.e., by the linear maps $D: \mathfrak{g} \rightarrow \mathfrak{g}$ such that

$$
D[X, Y]=[D(X), Y]+[X, D(Y)], \quad X, Y \in \mathfrak{g}
$$

A derivation $D$ will be called inner if there exists an element $X \in \mathfrak{g}$ such that $D(Y)=\operatorname{ad}(X)(Y):=$ $[X, Y]$ for all $Y \in \mathfrak{g}$. Otherwise, it will be called an outer derivation.

Definition 1. Let $\mathfrak{g}$ be a Lie algebra of dimension d. An external torus of derivations is an Abelian subalgebra of $\operatorname{Der}(\mathfrak{g})$, the generators of which are semisimple.

Due to the semi-simplicity and Abelianity, the maps $f \otimes_{\mathbb{R}} \operatorname{Id} \in \operatorname{End}\left(\mathfrak{g} \otimes_{\mathbb{R}} \mathbb{C}\right)$ are simultaneously diagonalizable over the complex field $\mathbb{C}$. For complex Lie algebras, maximal tori $t$ are known to be conjugate to each other, while for the real case the maximal tori are divided into a finite number of equivalence classes [25]. In both cases, the dimension of a maximal torus is a scalar invariant $r(\mathfrak{g})$ of the Lie algebra, called the rank of $\mathfrak{g}$.

One important structural result (see, e.g., [26] and references therein) states that any real or complex solvable Lie algebra $r$ admits a decomposition

$$
\mathfrak{r}=\mathfrak{t} \vec{\oplus} \mathfrak{n}
$$

satisfying the relations

$$
[\mathfrak{t}, \mathfrak{n}] \subset \mathfrak{n},[\mathfrak{n}, \mathfrak{n}] \subset \mathfrak{n},[\mathfrak{t}, \mathfrak{t}] \subset \mathfrak{n},
$$

where $\mathfrak{n}$ is the maximal nilpotent ideal of $\mathfrak{r}$ (called the nilradical) and $\vec{\oplus}$ denotes the semidirect sum determined by the complementary linear space $t$ formed by linearly nil-independent outer derivations of $\mathfrak{n}$. It can be further shown that the dimension of $\mathfrak{t}$ satisfies the following inequality

$$
\operatorname{dim} \mathfrak{n}-\operatorname{dim}[\mathfrak{n}, \mathfrak{n}] \geq \operatorname{dim} \mathfrak{t}
$$

providing an upper bound for the rank of a solvable Lie algebra.

\subsection{Cohomologically Rigid Lie Algebras}

Let $\mathcal{L}^{n}$ denote the variety of $n$-dimensional real Lie algebras $\mathfrak{g}=\left(\mathbb{R}^{n},[,]_{\mathfrak{g}}\right)$ over $\mathbb{R}$. The general linear group $G L(n, \mathbb{R})$ acts naturally on $\mathcal{L}^{n}$ by means of:

$$
(f \star \mathfrak{g})(X, Y)=f\left(\left[f^{-1}(X), f^{-1}(Y)\right]_{\mathfrak{g}}\right), f \in G L(n, \mathbb{K}), X, Y \in \mathfrak{g}
$$


The orbit $\mathcal{O}(\mathfrak{g})$ of a Lie algebra $\mathfrak{g}$ is thus given by all algebras isomorphic to $\mathfrak{g}$. The space $\mathcal{O}(\mathfrak{g})$ can further be identified with the homogeneous space $G L(n, \mathbb{R}) / \operatorname{Aut}(\mathfrak{g})$, from which the identity $\operatorname{dim} \mathcal{O}(\mathfrak{g})=n^{2}-\operatorname{dim} \operatorname{Der}(\mathfrak{g})$ follows at once.

Definition 2. A Lie algebra $\mathfrak{g}$ is called rigid if the orbit $\mathcal{O}(\mathfrak{g})$ is an open set on the space of structure constants $\mathbb{R}^{n^{3}}$ with respect to the Euclidean topology.

An equivalent definition can be obtained using the openness of the orbit $\mathcal{O}(\mathfrak{g})$ with respect to the Zariski topology of $\mathcal{L}^{n}[1,27]$. Although rigidity is primarily a topological notion, sufficient conditions to ensure that a Lie algebra is rigid can be obtained by purely algebraic means, using the Chevalley cohomology [3]. This approach involves the computation of the cohomology spaces $H^{p}(\mathfrak{g}, \mathfrak{g})$ for $p \leq 3$. The spaces $H^{0}(\mathfrak{g}, \mathfrak{g})$ and $H^{1}(\mathfrak{g}, \mathfrak{g})$ are identified with the center $Z(\mathfrak{g})$ and the outer derivations $\operatorname{Der}(\mathfrak{g}) / \operatorname{IDer}(\mathfrak{g})$ of $\mathfrak{g}$, respectively [6]. To illustrate how the rigidity problem leads naturally to cohomological methods, we recall the notion of contraction of Lie algebras (see, e.g., [28-31] and references therein).

Let $\mathfrak{g}$ be a Lie algebra and $\Phi_{\varepsilon} \in A u t(\mathfrak{g})$ a family of automorphisms of $\mathfrak{g}$, where $\varepsilon \in(0,1]$ with $\Phi_{1}$ being the identity. For any $X, Y \in \mathfrak{g}$ we define

$$
[X, Y]_{\Phi_{\varepsilon}}:=\Phi_{\varepsilon}^{-1}\left[\Phi_{\varepsilon}(X), \Phi_{\varepsilon}(Y)\right],
$$

which are obviously the brackets of the Lie algebra over the transformed basis. Now, suppose that the limit

$$
[X, Y]_{0}:=\lim _{\varepsilon \rightarrow 0} \Phi_{\varepsilon}^{-1}\left[\Phi_{\varepsilon}(X), \Phi_{\varepsilon}(Y)\right]
$$

exists for any $X, Y \in \mathfrak{g}$. Then, Equation (6) defines a Lie algebra $\mathfrak{g}^{\prime}$ called the contraction of $\mathfrak{g}$ (by $\left.\Phi_{\varepsilon}\right)$, non-trivial if $\mathfrak{g}$ and $\mathfrak{g}^{\prime}$ are non-isomorphic, and trivial otherwise. A contraction for which there exists some basis $\left\{Y_{1}, . ., Y_{n}\right\}$ such that the contraction matrix $A_{\Phi}$ is diagonal, i.e., adopts the form

$$
\left(A_{\Phi}\right)_{i j}=\delta_{i j} \varepsilon^{n_{j}}, \quad n_{j} \in \mathbb{R}, \varepsilon>0,
$$

is further known as a generalized Inönü-Wigner contraction [31].

Deformations of Lie algebras, deeply related to contractions and cohomology [4,5], originally arise from the study of local geometric properties of the variety $\mathcal{L}^{n}$ when considered as a transformation space. In this context, a formal deformation $\mathfrak{g}_{t}$ of a Lie algebra $\mathfrak{g}=(V, \mu)$ is given by the deformed commutator:

$$
[X, Y]_{\varepsilon}:=[X, Y]+\psi_{m}(X, Y) \varepsilon^{m}
$$

where $\varepsilon$ is a parameter and $\psi_{m}: V \times V \rightarrow V$ is a skew-symmetric bilinear map. Imposing the Jacobi identity (up to quadratic order of $\varepsilon$ ) to the deformed commutator, we obtain:

$$
\begin{gathered}
{\left[X_{i},\left[X_{j}, X_{k}\right]_{\varepsilon}\right]_{\varepsilon}+\left[X_{k},\left[X_{i}, X_{j}\right]_{\varepsilon}\right]_{\varepsilon}+\left[X_{j},\left[X_{k}, X_{i}\right]_{\varepsilon}\right]_{\varepsilon}=\varepsilon d \psi_{1}\left(X_{i}, X_{j}, X_{k}\right)+} \\
\varepsilon^{2}\left(\frac{1}{2}\left[\psi_{1}, \psi_{1}\right]+d \psi_{2}\right)\left(X_{i}, X_{j}, X_{k}\right)+\mathcal{O}\left(\varepsilon^{3}\right),
\end{gathered}
$$

where

$$
\begin{gathered}
d \psi_{l}\left(X_{i}, X_{j}, X_{k}\right):=\left[X_{i}, \psi_{l}\left(X_{j}, X_{k}\right)\right]+\left[X_{k}, \psi_{l}\left(X_{i}, X_{j}\right)\right]+\left[X_{j}, \psi_{l}\left(X_{k}, X_{i}\right)\right]+ \\
\psi_{l}\left(X_{i},\left[X_{j}, X_{k}\right]\right)+\psi_{l}\left(X_{k},\left[X_{i}, X_{j}\right]\right)+\psi_{l}\left(X_{j},\left[X_{k}, X_{i}\right]\right), \quad l=1,2
\end{gathered}
$$

and

$$
\frac{1}{2}\left[\psi_{1}, \psi_{1}\right]\left(X_{i}, X_{j}, X_{k}\right):=\psi_{1}\left(\psi_{1}\left(X_{i}, X_{j}\right), X_{k}\right)+\psi_{1}\left(\psi_{1}\left(X_{j}, X_{k}\right), X_{i}\right)+\psi_{1}\left(\psi_{1}\left(X_{k}, X_{i}\right), X_{j}\right) .
$$


To satisfy the Jacobi identity, the conditions

$$
\begin{array}{r}
d \psi_{1}\left(X_{i}, X_{j}, X_{k}\right)=0, \\
\frac{1}{2}\left[\psi_{1}, \psi_{1}\right]\left(X_{i}, X_{j}, X_{k}\right)+d \psi_{2}\left(X_{i}, X_{j}, X_{k}\right)=0
\end{array}
$$

must be fulfilled. The expression in Equation (10) satisfied by $\psi_{1}$ characterizes it as a 2-cocycle in the second cohomology space $H^{2}(\mathfrak{g}, \mathfrak{g})$, while Equation (11) implies that the deformation has to satisfy an integrability condition (further constraints are obtained if the deformed bracket is developed up to higher order; see, e.g., [28]). The latter is given by the condition

$$
\frac{1}{2}[\varphi, \varphi]\left(X_{i}, X_{j}, X_{k}\right)=0, \quad \forall X_{i}, X_{j}, X_{k} \in \mathfrak{g} .
$$

Hence, if $\varphi$ is an integrable cocyle, the linear deformation given by

$$
[X, Y]_{\varepsilon}:=[X, Y]+\varepsilon \varphi(X, Y)
$$

satisfies the Jacobi identity and defines a Lie algebra. In particular, nullity of $H^{2}(\mathfrak{g}, \mathfrak{g})$ implies that any deformation is isomorphic to $\mathfrak{g}$. This constitutes one possible formulation of the Nijenhuis-Richardson criterion, from which ample classes of Lie algebras such as semisimple and parabolic algebras are easily shown to be rigid $[17,18,20,32]$.

Proposition 1. Let $\mathfrak{g}$ be a Lie algebra. If the condition $\operatorname{dim} H^{2}(\mathfrak{g}, \mathfrak{g})=0$ holds, then $\mathfrak{g}$ is rigid.

Lie algebras satisfying $H^{2}(\mathfrak{g}, \mathfrak{g})=0$ are usually called cohomologically rigid. However, as was also shown in [17], the nullity of the cohomology space $H^{2}(\mathfrak{g}, \mathfrak{g})$ is not a necessary condition for rigidity, i.e., there exist Lie algebras satisfying $H^{2}(\mathfrak{g}, \mathfrak{g}) \neq 0$ but possessing an open orbit $[19,20]$. Such Lie algebras, called geometrically rigid, are lesser known and harder to obtain than cohomologically rigid algebras. Cohomologically rigid Lie algebras (over $\mathbb{R}$ and $\mathbb{C}$ ) are dominant in low dimensions and certain types of maximal tori [16,33-35], although for dimensions $n \geq 12$ continuous series of geometrically (solvable) rigid Lie algebras have been found $[12,13,15,17,24,36,37]$. In this latter case, the analysis of the cohomology space $H^{3}(\mathfrak{g}, \mathfrak{g})$, based on the so-called Rim map defined in Equation (9), has been shown to be of crucial importance [19,38].

The main tool in the cohomological approach is the Hochschild-Serre factorization theorem [3], a procedure that simplifies the explicit computation of the cohomology classes. For the purpose of this work, it suffices to restrict ourselves to the case of solvable real Lie algebras $\mathfrak{r}=\mathfrak{t} \vec{\oplus} \mathfrak{n}$ such that $\mathfrak{t}$ is Abelian and the operators $\operatorname{ad}_{\mathfrak{r}} T(T \in \mathfrak{t})$ are diagonal. The symbol $\vec{\oplus}$ indicates that the sum is semidirect, with $\mathfrak{t}$ acting on $\mathfrak{n}$ by derivations. In these conditions (see, e.g., [6]), the cohomology space $H^{p}(\mathfrak{r}, \mathfrak{r})$ satisfies the isomorphism

$$
H^{p}(\mathfrak{r}, \mathfrak{r}) \simeq \sum_{a+b=p} H^{a}(\mathfrak{t}, \mathbb{R}) \otimes H^{b}(\mathfrak{n}, \mathfrak{r})^{\mathfrak{t}},
$$

where the space of $\mathfrak{t}$-invariant cocycle classes of $\mathfrak{n}$ with values in $\mathfrak{r}$ is defined as

$$
H^{b}(\mathfrak{n}, \mathfrak{r})^{\mathfrak{t}}=\left\{[\varphi] \in H^{b}(\mathfrak{n}, \mathfrak{r}) \mid(T . \varphi)=0, T \in \mathfrak{t}\right\} .
$$

The invariance condition of a $b$-cochain $\varphi$ is given by

$$
(T . \varphi)\left(Z_{1}, \cdots, Z_{b}\right)=\left[T, \varphi\left(Z_{1}, \cdots, Z_{b}\right)\right]-\sum_{s=1}^{b} \varphi\left(Z_{1}, \cdots,\left[T, Z_{s}\right], \cdots, Z_{b}\right) .
$$


Using the well known fact that $H^{a}(\mathfrak{t}, \mathbb{R})=\bigwedge^{a} \mathfrak{t}$, it is straightforward to verify that $H^{p}(\mathfrak{r}, \mathfrak{r})=0$ holds if and only if $H^{b}(\mathfrak{n}, \mathfrak{r})^{\mathfrak{t}}=0$ for $0 \leq b \leq p$.

We observe that, if a complex Lie algebra $\mathfrak{g}^{\prime}$ admits the real form $\mathfrak{g}$, i.e., such that $\mathfrak{g}^{\prime} \simeq \mathfrak{g} \otimes_{\mathbb{R}} \mathbb{C}$, then it follows from the Chevalley cohomology that [33]

$$
\operatorname{dim}_{\mathbb{R}} H^{2}(\mathfrak{g}, \mathfrak{g})=\operatorname{dim}_{\mathbb{C}} H^{2}\left(\mathfrak{g}^{\prime}, \mathfrak{g}^{\prime}\right) .
$$

In this sense, a real Lie algebra $\mathfrak{g}$ is cohomologically rigid if

$$
\operatorname{dim}_{\mathbb{R}} H^{2}(\mathfrak{g}, \mathfrak{g})=0 .
$$

Clearly, in these conditions, the complexified algebra $\mathfrak{g}^{\prime}=\mathfrak{g} \otimes_{\mathbb{R}} \mathbb{C}$ is a complex cohomologically rigid Lie algebra. Conversely, real forms $\mathfrak{g}_{i}$ of a complex cohomologically rigid Lie algebra $\mathfrak{g}^{\mathbb{C}}=\mathfrak{g}_{i} \otimes \mathbb{C}$ are cohomologically rigid. It should however be observed that there exist purely complex cohomologically rigid Lie algebras that do not admit real forms [21]. A negative test for a solvable Lie algebra $\mathfrak{r}$ to be cohomologically rigid is further given by the following relation (see, e.g., [27,39]):

$$
\operatorname{dim} \operatorname{Der}(\mathfrak{r}) \leq \operatorname{dim} \mathfrak{r}+\operatorname{dim} H^{2}(\mathfrak{r}, \mathfrak{r}) .
$$

It follows that if $\operatorname{dim} \operatorname{Der}(\mathfrak{r})>\operatorname{dim} \mathfrak{r}$, then $\mathfrak{r}$ cannot be cohomologically rigid. In particular, if a real (or complex) Lie algebra satisfying the decomposition (1) is rigid, an important structural result of R. Carles implies that $\mathfrak{t}$ must be a maximal external torus of derivations of $\mathfrak{n}$ [27].

\section{Rigid Lie Algebras with Filiform Nilradical}

For studying nilpotent Lie algebras, we recall an extremely useful invariant called the characteristic sequence $c(\mathfrak{n})$ of $\mathfrak{n}$. Given a nilpotent Lie algebra $\mathfrak{n}$, for a non-zero element $X \in \mathfrak{n} \backslash[\mathfrak{n}, \mathfrak{n}]$, we consider the decreasing sequence of dimensions of the Jordan blocks of the linear operator $\operatorname{ad}(X)$.

$$
c(X)=\left(c_{1}(X), c_{2}(X), \cdots, c_{k}(X), 1\right), \quad c_{i}(X) \geq c_{i+1}(X) \geq 1 .
$$

Definition 3. The characteristic sequence of a nilpotent Lie algebra $\mathfrak{n}$ is defined as

$$
c(\mathfrak{n})=\sup \{c(X) \mid X \in \mathfrak{n} \backslash[\mathfrak{n}, \mathfrak{n}]\} .
$$

In particular, if a nilpotent Lie algebra has characteristic sequence $c(\mathfrak{n})=(n-1,1)$, then there exists a basis $\left\{X_{1}, \cdots, X_{n}\right\}$ of $\mathfrak{n}$ such that

$$
\left[X_{1}, X_{j}\right]=X_{j+1}, 2 \leq j \leq n-1 .
$$

Such Lie algebras are commonly called filiform [22]. The remaining commutators $\left[X_{i}, X_{j}\right]$ for $2 \leq i, j$ are related by means of the Jacobi condition

$$
\left[X_{1},\left[X_{i}, X_{j}\right]\right]+\left[X_{j},\left[X_{1}, X_{i}\right]\right]+\left[X_{i},\left[X_{j}, X_{1}\right]\right]=0 .
$$

We observe that, as a consequence of the upper bound (Equation (3)), a filiform Lie algebra has a maximal torus of dimension at most two. It is shown in [22] that there exist only two isomorphism classes (called the model filiform algebras) possessing rank two, and given, respectively, by

- $\quad L_{n}(n \geq 3)$ :

$$
\left[X_{1}, X_{j}\right]=X_{j+1}, 2 \leq j \leq n-1
$$

- $Q_{n}(n=2 q \geq 6)$ :

$$
\left[X_{1}, X_{i}\right]=X_{i+1}, \quad\left[X_{j}, X_{n+1-j}\right]=(-1)^{j} X_{n}, \quad 2 \leq i \leq n-2,2 \leq j \leq q .
$$


If $\mathfrak{t}_{L}$ and $\mathfrak{t}_{Q}$ denote a maximal torus of $L_{n}$ and $Q_{n}$, respectively, it can be easily shown that the eigenvalue spectrum of their tori is given by

$$
\begin{aligned}
\operatorname{spec}\left(\mathfrak{t}_{L}\right) & =(\lambda, \mu, \lambda+\mu, 2 \lambda+\mu, \cdots,(n-3) \lambda+\mu,(n-2) \lambda+\mu), \\
\operatorname{spec}\left(\mathfrak{t}_{Q}\right) & =(\lambda, \mu, \lambda+\mu, 2 \lambda+\mu, \cdots,(n-3) \lambda+\mu,(n-3) \lambda+2 \mu),
\end{aligned}
$$

where $\lambda, \mu$ are integers. The remaining filiform Lie algebras are all of rank one, and the generic properties of their tori have been studied in [40]. In particular, it is shown that any $n$-dimensional filiform Lie algebra of rank one is isomorphic to one of the following Lie algebras (in [40], a third type $C_{n}^{k}$ is given, which must be discarded, as it actually has rank two and leads to the model algebra $Q_{n}$ ):

1. $A_{n}^{k}\left(\lambda_{1}, \cdots, \lambda_{l-1}\right), l=\left[\frac{n+1-k}{2}\right], 2 \leq k \leq n-3$ :

$$
\begin{array}{ll}
{\left[X_{1}, X_{i}\right]=X_{i+1},} & 2 \leq i \leq n-1 \\
{\left[X_{i}, X_{i+1}\right]=\lambda_{i-1} X_{2 i+k-1},} & 2 \leq i \leq l \\
{\left[X_{i}, X_{j}\right]=a_{i j} X_{i+j+k-2},} & 2 \leq i<j, i+j+k \leq n
\end{array}
$$

2. $\quad B_{n}^{k}\left(\lambda_{1}, \cdots, \lambda_{l-1}\right), n=2 m, l=\left[\frac{n-k}{2}\right], 2 \leq k \leq n-3$ :

$$
\begin{array}{ll}
{\left[X_{1}, X_{i}\right]=X_{i+1},} & 2 \leq i \leq n-2 \\
{\left[X_{i}, X_{n+1-i}\right]=(-1)^{i} X_{n},} & 2 \leq i \leq n-1 \\
{\left[X_{i}, X_{i+1}\right]=\lambda_{i-1} X_{2 i+k-1},} & 2 \leq i \leq l \\
{\left[X_{i}, X_{j}\right]=a_{i j} X_{i+j+k-2,},} & 2 \leq i<j, i+j+k \leq n-2
\end{array}
$$

The parameters $\left(\lambda_{1}, \cdots, \lambda_{l-1}\right)$ are non-simultaneously vanishing and satisfy the Jacobi relations. Moreover, the constants $a_{i, j}$ satisfy the conditions

$$
a_{i, j}=a_{i, j+1}+a_{i+1, j}, a_{i, i+1}=\lambda_{i-1} .
$$

Now, let $\mathfrak{n}$ be isomorphic to either $A_{n}^{k}$ or $B_{n}^{k}$ and consider the endomorphism $F: \mathfrak{n} \rightarrow \mathfrak{n}$ defined by

$$
X_{1}^{\prime}=X_{1}, X_{j}^{\prime}=\varepsilon X_{j}, X_{n}^{\prime}=\varepsilon^{2} X_{n}, 2 \leq j \leq n-1 .
$$

Computing the brackets over the transformed basis, we obtain the relations

$$
\left[X_{1}^{\prime}, X_{j}^{\prime}\right]=X_{j+1}^{\prime},\left[X_{i}^{\prime}, X_{i+1}^{\prime}\right]=\varepsilon \lambda_{i-1} X_{2 i+k-1}^{\prime},\left[X_{i}^{\prime}, X_{j}^{\prime}\right]=a_{i j} \varepsilon X_{i+j+k-2}^{\prime}
$$

for both cases, and

$$
\left[X_{i}^{\prime}, X_{n+1-i}^{\prime}\right]=(-1)^{i} X_{n}^{\prime}
$$

for the $B_{n}^{k}$ series. For the limit $\varepsilon \rightarrow 0$, it follows that $A_{n}^{k}$ contracts onto the model filiform Lie algebra $L_{n}$, while $B_{n}^{k}$ contracts onto $Q_{n}$. In this context, it is convenient to separate the rigidity analysis of rank one solvable Lie algebras with filiform nilradical into the two cases, depending whether the nilradical belongs to the $A_{n}^{k}$ or $B_{n}^{k}$ class, although some conclusions can be obtained simultaneously for both types (see [15]). We mention that the so-called Bratzlavsky series [36], the first of the solvable geometrically rigid Lie algebras to be described in detail [37], has a nilradical of type $A_{n}^{k}$. Other large classes of rigid Lie algebras based on this nilpotent algebra have been obtained for the values $k=2,3,4$, covered in full detail (see $[15,24,37]$ and references therein), as well as in the (full) classification of solvable rigid algebras in low dimensions [16].

In the sequel, we are principally interested in rank one cohomologically rigid Lie algebras with nilradical of type $B_{n}^{k}$. In this context, it is well known that, for any integer $k \geq 2$, 
the $n=\left(k+4+\frac{1-(-1)^{k}}{2}\right)$-dimensional real nilpotent Lie algebra $\mathfrak{n}_{n}^{k}$ defined over a basis $\left\{X_{1}, \cdots, X_{n}\right\}$ by the commutators

$$
\begin{array}{ll}
{\left[X_{1}, X_{j}\right]=X_{j+1},} & 2 \leq j \leq n-2 \\
{\left[X_{i}, X_{n+1-i}\right]=(-1)^{i} X_{n},} & 2 \leq i \leq \frac{n}{2} \\
{\left[X_{2}, X_{3}\right]=X_{3+k},} & {\left[X_{2}, X_{4}\right]=\frac{1-(-1)^{k}}{2} X_{4+k} .}
\end{array}
$$

is cohomologically rigid (this is immediate from the results in [15]). A short computation shows that the spectrum corresponding to these algebras is given by

$$
\operatorname{spec}(\mathfrak{t})=(1, k, k+1, \cdots, n+k-3, n+2 k-3),
$$

and actually corresponds to the lowest possible dimension for which the condition $C_{23}^{3+k} \neq 0$ is satisfied. It may seem surprising that, by merely adding one or two additional commutators (depending on the parity of $k$ ), we obtain a rigid Lie algebra of rank one. This fact suggests that the number of (cohomologically) rigid Lie algebras of rank one and possessing a torus $\mathfrak{t}$ with the spectrum in Equation (28) must be very ample. We see that large families of rigid Lie algebras with vanishing cohomology emerge from this eigenvalue spectrum. The range of dimensions for which such algebras exist are shown to be determined by the value and the parity of $k$.

\section{Cohomologically Rigid Rank One Lie Algebras with Given Eigenvalue Spectrum}

In this section, we analyze some generic features of real solvable Lie algebras $\mathfrak{r}_{n}^{k}=\mathfrak{t} \vec{\oplus} \mathfrak{n}_{n}^{k}$ of rank one, the torus $\mathfrak{t}$ of which has eigenvalues $\operatorname{spec}(\mathfrak{t})=(1, k, k+1, \cdots, n+k-3, n+2 k-3)$ and such that the nilradical contracts $\mathfrak{n}_{n}^{k}$ onto the model filiform algebra $Q_{n}$. Let $\left\{T, X_{1}, X_{2}, \ldots, X_{n}\right\}$ be a basis of $\mathfrak{r}_{n}^{k}$. The action of $T$ over the nilradical is given by

$$
\left[T, X_{1}\right]=X_{1},\left[T, X_{j}\right]=(k+j-2) X_{j},\left[T, X_{n}\right]=(n+2 k-3) X_{n}, \quad(2 \leq j \leq n-1) .
$$

As follows from this action, for any $i, j \geq 1$, the commutator $\left[X_{i}, X_{j}\right]$ corresponds to an element of $\mathfrak{n}_{n}^{k}$ with eigenvalue $2 k+i+j-4$, from which we conclude that the commutators adopt the generic form

$$
\begin{array}{r}
{\left[X_{1}, X_{j}\right]=C_{1, j}^{j+1} X_{j+1}, 2 \leq j \leq n-2,} \\
{\left[X_{i}, X_{j}\right]=C_{i, j}^{i+j+k-2} X_{i+j+k-2}, i+j<n+1-k,} \\
{\left[X_{i}, X_{n+1-i}\right]=C_{i, n+1-i}^{n} X_{n}, 2 \leq i \leq \frac{n}{2} .}
\end{array}
$$

In particular, as we require that $\left[X_{2}, X_{n-1}\right] \neq 0$ holds, it can be immediately verified by the Jacobi condition that $C_{i, n+1-i}^{n}=(-1)^{i} C_{2, n-1}^{n}$ is satisfied for all $i \geq 2$. Without loss of generality, we can assume that $C_{2, n-1}^{n}=1$, so that the generic structure is given by

$$
\begin{array}{ll}
{\left[X_{1}, X_{j}\right]=X_{j+1},} & 2 \leq j \leq n-2, \\
\left.X_{i}, X_{j}\right]=C_{i, j}^{i+j+k-2} X_{i+j+k-2}, & i+j<n+1-k, \\
{\left[X_{i}, X_{n+1-i}\right]=(-1)^{i} X_{n},} & 2 \leq i \leq \frac{n}{2} .
\end{array}
$$

The Lie algebras of the type in Equation (31) are therefore determined by the solutions to the polynomial equations defined by the Jacobi conditions for $1 \leq i \leq j \leq \ell \leq n-1$. The Jacobi condition applied to the triple $\left\{X_{1}, X_{i}, X_{j}\right\}$ with $i<j$ leads to the linear system

$$
C_{i, j}^{i+j+k-2}-C_{i+1, j}^{i+j+k-1}-C_{i, j+1}^{i+j+k-1}=0,2 \leq i<j .
$$


In particular, for $j=i+1$, we get the relations

$$
C_{i, i+1}^{2 i+k-1}-C_{i, i+2}^{2 i+k}=0, i \geq 2 .
$$

Introducing the notation $\alpha_{i-1}=C_{i, i+1}^{2 i+k-1}$ for $2 \leq i \leq\left[\frac{n-k}{2}\right]$, a cumbersome but routine computation shows that the system in Equation (32) admits the solution

$$
C_{i, j}^{i+j+k-2}=\sum_{p=1}^{\left[\frac{j+1-i}{2}\right]}(-1)^{p+1} \frac{\Gamma(j+1-i-p)}{(p-1) ! \Gamma(j+2-2 p-i)} \alpha_{i+p-2},
$$

where $\Gamma(z)$ denotes the Gamma function. With this reduction, any Lie algebra of the type in (31) will be characterized by the values $\alpha_{s}$ for $1 \leq s \leq\left[\frac{n-k-1}{2}\right]$. It remains to evaluate the Jacobi conditions for the triples $\left\{X_{i}, X_{j}, X_{l}\right\}$ with $i, j, l \geq 2$. Expanding these relations, we obtain the equations

$$
C_{j, l}^{j+l+\kappa-2} C_{i, j+l+\kappa-2}^{i+j+l+2 \kappa-4}-C_{i, j}^{i+j+\kappa-2} C_{i+j+\kappa-2, l}^{i+j+l+2 \kappa-4}-C_{i, l}^{i+l+\kappa-2} C_{j, i+l+\kappa-2}^{i+j+l+2 \kappa-4}=0 .
$$

We observe that, whenever the numerical relation

$$
i+j+l=n+3-\kappa
$$

is satisfied, the corresponding equation (Equation (35)) is linear, because it involves commutators of the type $\left[X_{a}, X_{n+1-a}\right]=(-1)^{a} X_{n}$. Now, replacing the $C_{j, l}^{j+l+\kappa-2}$ by the expression given by Equation (34), the system in Equation (35) reduces to a set of linear or homogeneous quadratic polynomials in the variables $\alpha_{s}$, so that the nilradical $\mathfrak{n}_{n}^{k}$ is completely specified (using the previous identities) by the "diagonal" sequence

$$
\left(\alpha_{1}, \ldots, \alpha_{p_{0}}\right)=\left(C_{2,3}^{k+3}, \cdots, C_{p_{0}, p_{0}+1}^{n-1-\frac{1+(-1)^{k}}{2}}\right), p_{0}=\left[\frac{n-k-1-\frac{1+(-1)^{k}}{2}}{2}\right] .
$$

At this point, is is worthy to be mentioned that a kind of systematization to the solution analysis of Equation (35) for given values of $k$ and $n$ can be obtained if we proceed as follows: For each $s \geq 1$, we define $q_{0}=p_{0}-s$ and evaluate Equation (35) for the sequence

$$
\beta_{s}=\left(\beta_{1}, \cdots, \beta_{p_{0}}\right)=\left(0, \cdots, 0, \alpha_{p_{0}-s}, \cdots, \alpha_{p_{0}}\right) .
$$

We can assume that $\alpha_{p_{0}-s} \neq 0$, and thus we can normalize it to one. This avoids repetitions of tuples when increasing the value of $s$. We then analyze whether the system in Equation (35), when reduced with respect to the sequence in Equation (37), admits a solution for the parameters $\left\{\alpha_{p_{0}-s+1}, \cdots \alpha_{p_{0}}\right\}$. For each of the sequences $\beta_{s}$, the Jacobi conditions (possibly) simplify and provide the solutions of a given type. This simplification is specially useful for high dimensions, where a great number of (parameterized) solutions may exist, solving the system in Equation (35) directly may be very time consuming.

Although for low values of $k$ and $n$ such a subdivision may not be required as these equations can be solved by hand, for increasing dimensions $k+6 \leq n \leq 4 k$, the use of computer methods becomes a necessity to obtain the solutions for the quadratic equations determined by Equation (35), as well as to compute the cohomology $H^{2}\left(\mathfrak{r}_{n}^{k}, \mathfrak{r}_{n}^{k}\right)$. It is therefore unavoidable, from a certain dimension onwards, to use computer packages to determine precise (even partial) classifications of rigid Lie algebras.

The computation of the cohomologies has been done using the symbolic computation package SuperLie (see [41,42] and references therein) as well as specific codes for rank one Lie algebras developed by the authors, which have also been adapted to solve the quadratic systems given by the Jacobi 
conditions. Both programs have been executed on different platforms in order to double-check the cohomologies.

\section{A Case Study: $k \leq 5$}

For low values $k=2,3,4,5$, the analysis of the Jacobi conditions in Equation (35) can still be developed directly, due to the low dimension of the resulting algebras and the relative simplicity of the Jacobi conditions. It is therefore instructive to consider these values in detail, even if most of the rigid algebras that arise in these cases have already been considered in the literature (see, for instance, the references in $[13,15,16,33])$. The dimensions analyzed are $n \geq k+4$ or $k+5$ depending on the parity of $k$, as the series given in Equation (27) describes the lowest-dimensional case for which $C_{2,3}^{k+3} \neq 0$ is satisfied.

4.1. $k=2$

Let $\mathfrak{r}_{n}^{2}=\mathfrak{t} \vec{\oplus} \mathfrak{n}_{n}^{2}$. For this value, the corresponding eigenvalue spectrum is given by

$$
\operatorname{spec}(\mathfrak{t})=(1,2,3, \cdots, n-1, n+1)
$$

We assume that $n>k+4$, as $n=k+4=6$ is already covered by Equation (27).

1. $\operatorname{dim} \mathfrak{n}=8$. There is only one solution, corresponding to $\left(\alpha_{1}, \alpha_{2}\right)=(1,-2)$. A short computation shows that $H^{2}\left(\mathfrak{r}_{8}^{2}, \mathfrak{r}_{8}^{2}\right)=0$.

2. $\operatorname{dim} \mathfrak{n}=10$. The Jacobi conditions in Equation (35) reduce to the equations

$$
2 \alpha_{1}-\alpha_{2}-\alpha_{3}=0,2 \alpha_{2}^{2}-2 \alpha_{1} \alpha_{3}-\alpha_{2} \alpha_{3}=0 \text {. }
$$

There are only two solutions corresponding to the triples $\left(\alpha_{1}, \alpha_{2}, \alpha_{3}\right)=(1,1,1)$ and $(1,-1,3)$. Both Lie algebras are cohomologically rigid.

3. $\operatorname{dim} \mathfrak{n}=12$. In this case, the Jacobi conditions lead to the system

$$
2 \alpha_{1}-3 \alpha_{2}+\alpha_{4}=0,2 \alpha_{2}^{2}-2 \alpha_{1} \alpha_{3}-\alpha_{2} \alpha_{3}=0,4 \alpha_{2} \alpha_{3}-6 \alpha_{3}^{2}-\left(2 \alpha_{1}-\alpha_{2}-\alpha_{3}\right) \alpha_{4}=0
$$

It admits four independent solutions, two of which are complex conjugate and two real non-rational solutions given by

$$
\begin{aligned}
& \left(\alpha_{1}, \alpha_{2}, \alpha_{3}, \alpha_{4}\right)=\left(1, \frac{2}{3}(1 \pm i \sqrt{2}), \pm i \sqrt{2}, \pm 2 i \sqrt{2}\right) \\
& \left(\alpha_{1}, \alpha_{2}, \alpha_{3}, \alpha_{4}\right)=\left(1, \pm \sqrt{\frac{2}{5}}, \frac{1}{15}(10 \mp \sqrt{10}), \frac{1}{5}(-10 \pm 3 \sqrt{10})\right)
\end{aligned}
$$

All these Lie algebras can be verified to have a vanishing second cohomology space, hence they are cohomologically rigid.

4. For $n=2 q \geq 14$, the Jacobi conditions have only the trivial solution $\alpha_{i}=0$, implying that there are no rank one solvable Lie algebras with the eigenvalues in Equation (38).

We remark that the existence of complex nonreal rigid Lie algebras is announced in [21], where the first examples are also given.

4.2. $k=3$

If $n=8$, the Lie algebra is rigid by Equation (27). 
1. For $\operatorname{dim} \mathfrak{n}=10$, the Jacobi conditions are trivially satisfied for any values of the variables $\alpha_{1}$ and $\alpha_{2}$, hence the Lie algebra is not rigid.

2. $\operatorname{dim} \mathfrak{n}=12$. The Jacobi conditions reduce to the equations:

$$
2 \alpha_{2}+\alpha_{3}=0,4 \alpha_{2}^{2}-3 \alpha_{1} \alpha_{3}-3 \alpha_{2} \alpha_{3}=0,
$$

admitting the two independent solutions $\left(\alpha_{1}, \alpha_{2}, \alpha_{3}\right)=(1,0,0)$ and $\left(1,-\frac{3}{5}, \frac{6}{5}\right)$. Both resulting solvable Lie algebras are cohomologically rigid.

3. $\operatorname{dim} \mathfrak{n}=14$. Three quadratic equations are obtained:

$$
2 \alpha_{2}-\alpha_{3}-\alpha_{4}=0,4 \alpha_{2}^{2}-3 \alpha_{1} \alpha_{3}-3 \alpha_{2} \alpha_{3}=0,10 \alpha_{3}^{2}+3 \alpha_{1} \alpha_{4}-5 \alpha_{2} \alpha_{3}-2 \alpha_{2} \alpha_{4}-4 \alpha_{3} \alpha_{4}=0 .
$$

There are four solutions, corresponding to the values

$$
\left(\alpha_{1}, \alpha_{2}, \alpha_{3}, \alpha_{4}\right)=(1,0,0,0),(1,3,3,3),\left(1, \frac{3}{4}, \frac{3}{7}, \frac{15}{14}\right)\left(1,-\frac{3}{7}, \frac{3}{7},-\frac{9}{7}\right) .
$$

All the resulting solvable Lie algebras are verified to have a vanishing second cohomology space, from which their cohomological rigidity follows.

4. For $\operatorname{dim} \mathfrak{n}=16$, the Jacobi conditions lead to five independent quadratic equations

$$
\begin{array}{r}
2 \alpha_{2}-3 \alpha_{3}+\alpha_{5}=0,4 \alpha_{2}^{2}-3 \alpha_{1} \alpha_{3}-3 \alpha_{2} \alpha_{3}=0,10 \alpha_{3}^{2}+3 \alpha_{1} \alpha_{4}-5 \alpha_{2} \alpha_{3}-2 \alpha_{2} \alpha_{4}-4 \alpha_{3} \alpha_{4}=0 \\
5 \alpha_{3}^{2}-4 \alpha_{2} \alpha_{4}-6 \alpha_{3} \alpha_{4}+2 \alpha_{2} \alpha_{5}+\alpha_{3} \alpha_{5}=0,20 \alpha_{4}^{2}-3 \alpha_{1} \alpha_{5}+5 \alpha_{2} \alpha_{5}-6 \alpha_{2} \alpha_{4}-21 \alpha_{3} \alpha_{4}-5 \alpha_{4} \alpha_{5}=0 .
\end{array}
$$

This system admits only two nontrivial solutions

$$
\left(\alpha_{1}, \alpha_{2}, \alpha_{3}, \alpha_{4}, \alpha_{5}\right)=(1,0,0,0,0),\left(1, \frac{3}{5}, \frac{3}{10}, 0,-\frac{3}{10}\right) .
$$

Again, the computation shows that the second cohomology space vanishes in both cases.

5. For $\operatorname{dim} \mathfrak{n}=18+2 \ell$ with $\ell \geq 0$, the only nontrivial solution to the Jacobi conditions is given by $\left(\alpha_{1}, \cdots, \alpha_{6+\ell}\right)=\left(1,0^{5+\ell}\right)$. This series is actually the analog of the so-called Bratzlavsky series (see, e.g., $[15,36,37])$ for nilradicals of type $B_{n}^{k}$.

Proposition 2. For any $n=18+2 \ell$ with $\ell \geq 0$, the solvable Lie algebra $\mathfrak{r}_{n}^{3}$ with nilradical corresponding to the solution $\left(\alpha_{1}, \cdots, \alpha_{6+\ell}\right)=\left(1,0^{5+\ell}\right)$ satisfies $\operatorname{dim} H^{2}\left(\mathfrak{r}_{n}^{3}, \mathfrak{r}_{n}^{3}\right)=0$.

One point that deserves a comment is the fact that all the rigid rank one solvable Lie algebras obtained for $k=3$ are rational, in contrast to the case of $k \neq 3$, where there always exist nonrational and even complex solutions.

4.3. $k=4$

Again, the case $n=8$ belongs to the family in Equation (27) and leads to a rigid Lie algebra.

1. For $\operatorname{dim} \mathfrak{n}=10$ there is only one nontrivial solution to the Jacobi conditions, given by $\left(\alpha_{1}, \alpha_{2}\right)=$ $(1,-2)$. The corresponding solvable Lie algebra has vanishing cohomology.

2. For $\operatorname{dim} \mathfrak{n}=12$, the Jacobi conditions are trivially satisfied for any values of the variables $\alpha_{1}$ and $\alpha_{2}$, hence the Lie algebra is not rigid.

3. For $\operatorname{dim} \mathfrak{n}=14$, we obtain parameterized families and no solutions with vanishing cohomology exist. 
4. For $\operatorname{dim} \mathfrak{n}=16$, we obtain four nontrivial solutions to the Jacobi conditions, two real and two complex ones, respectively:

$$
\begin{aligned}
\left(\alpha_{1}, \alpha_{2}, \alpha_{3}, \alpha_{4}, \alpha_{5}\right)= & \left(1, \frac{2}{105}( \pm 11 \mp \sqrt{226}+\sqrt{-1963+188 \sqrt{226}}), \frac{1}{63}(10+\sqrt{226} \mp \sqrt{577+62 \sqrt{226}})\right. \\
& \left.\frac{2}{63}(-10-\sqrt{226} \pm \sqrt{577+62 \sqrt{226}}), \frac{1}{9}(10+\sqrt{226} \mp \sqrt{-1057+122 \sqrt{226}})\right) \\
\left(\alpha_{1}, \alpha_{2}, \alpha_{3}, \alpha_{4}, \alpha_{5}\right)= & \left(1, \frac{2}{105}(11+\sqrt{226} \pm i \sqrt{1963+188 \sqrt{226}}), \frac{1}{63}(10-\sqrt{226} \pm i \sqrt{577+62 \sqrt{226}})\right. \\
& \left.\frac{2}{63}(-10+\sqrt{226} \mp i \sqrt{577+62 \sqrt{226}}), \frac{1}{9}(10-\sqrt{226} \mp i \sqrt{1057+122 \sqrt{226}})\right)
\end{aligned}
$$

The corresponding Lie algebras (complex and real) all satisfy the Richardson criterion and are cohomologically rigid.

5. For $\operatorname{dim} \mathfrak{n}=18$, the Jacobi conditions admit two nontrivial solutions

$$
\left(\alpha_{1}, \alpha_{2}, \alpha_{3}, \alpha_{4}, \alpha_{5}, \alpha_{6}\right)=\left(1, \frac{2}{5}, \frac{1}{5}, \frac{1}{5}, \frac{1}{5}, 0\right),\left(1,-1,-\frac{5}{3},-\frac{5}{3},-\frac{5}{3},-\frac{7}{3}\right)
$$

In addition, in this case, the corresponding solvable Lie algebras satisfy $H^{2}\left(\mathfrak{r}_{18}^{4}, \mathfrak{r}_{18}^{4}\right)=0$.

6. Finally, for any $n \geq 20$, the only solution to the system of Jacobi equations is given by $\alpha_{i}=0$, corresponding to the rank two model algebra $Q_{n}$. Hence, no rank one solvable algebras with the given eigenvalues exist.

4.4. $k=5$

As the last case to be explicitly analyzed for all dimensions, we consider $k=5$. By Equation (27), we already know that the nilradical in $n=10$ leads to a rigid Lie algebra. We show that, for this value of $k$, the same type of solution leads to solvable Lie algebras that are either cohomologically or geometrically rigid, depending on the dimension.

1. For $\operatorname{dim} \mathfrak{n}=12,14$ and 16 , we obtain parameterized families of nilpotent algebras that can be deformed into each other. No cohomologically rigid Lie algebras exist.

2. If $\operatorname{dim} \mathfrak{n}=18$, Equation (35) admits a two-parameter family of solutions, as well as two isolated solutions

$$
\left(\alpha_{1}, \alpha_{2}, \alpha_{3}, \alpha_{4}, \alpha_{5}\right)=\left(0,0,1, \frac{9}{5}, 3\right),\left(0,0,0,1,0, \frac{5}{24}\right) .
$$

The solvable Lie algebras corresponding to these values are not cohomologically rigid, as they both satisfy $\operatorname{dim} H^{2}\left(\mathfrak{r}_{18}^{5}, \mathfrak{r}_{18}^{5}\right)=1$. Moreover, they can be deformed into the family, so that they are not geometrically rigid.

3. The Jacobi conditions for $\operatorname{dim} \mathfrak{n}=20$ admit two nontrivial solutions

$$
\left(\alpha_{1}, \alpha_{2}, \alpha_{3}, \alpha_{4}, \alpha_{5}, \alpha_{6}\right)=(1,0,0,0,0),\left(1, \frac{5}{8}, \frac{5}{24}, 0,0, \frac{5}{24}\right) .
$$

The corresponding solvable Lie algebras $\mathfrak{r}_{20}^{5}$ satisfy in both cases $H^{2}\left(\mathfrak{r}_{20}^{5}, \mathfrak{r}_{20}^{5}\right)=0$.

4. For $\operatorname{dim} \mathfrak{n}=22$, we find two nontrivial solutions to Equation (35)

$$
\begin{aligned}
\left(\alpha_{1}, \alpha_{2}, \alpha_{3}, \alpha_{4}, \alpha_{5}\right)=\quad & (1,0,0,0,0,0), \\
& \left(1, \frac{5}{14}, \frac{5}{42}(5 \pm \sqrt{22}), \frac{5}{126}(17 \mp 4 \sqrt{22}), \frac{5}{126}(17 \mp 4 \sqrt{22}), \frac{5}{126}(19 \mp 5 \sqrt{22})\right) .
\end{aligned}
$$

In both cases, the solutions lead to cohomologically rigid Lie algebras. 
5. For $\operatorname{dim} \mathfrak{n}=24+4 l(l \geq 0)$, the only nontrivial solution is given by

$$
\left(\alpha_{1}, \ldots, \alpha_{7+2 l}\right)=(1,0, \ldots, 0) .
$$

If we compute the cohomology of the associated solvable Lie algebra $\mathfrak{r}_{24+4 l}^{5}$, we find that it does not vanish, but satisfies $\operatorname{dim} H^{2}\left(\mathfrak{r}_{24+4 l}^{5}, r_{24+4 l}^{5}\right)=1$. However, this Lie algebra is always geometrically rigid, as can be shown with a topological argument (see, for instance, the argument in [37], which is also valid for this case).

6. For $\operatorname{dim} \mathfrak{n}=26+4 l(l \geq 0)$, the only nontrivial solution is again

$$
\left(\alpha_{1}, \ldots, \alpha_{8+2 l}\right)=(1,0, \ldots, 0),
$$

but, in contrast to the previous case, we have that $\operatorname{dim} H^{2}\left(\mathfrak{r}_{26+4 l}^{5}, \mathfrak{r}_{26+4 l}^{5}\right)=0$, showing that the Lie algebras $\mathfrak{r}_{26+4 l}^{5}$ are cohomologically rigid.

As a consequence of this analysis we conclude that, depending on the parity of $k$, for high dimensions $n \geq 4 k$ we either obtain only the trivial solution for the equations determined by the Jacobi conditions (hence the non-existence of rank one Lie algebras with the prescribed eigenvalue spectrum) or the solution $(1,0, \ldots, 0)$, the cohomological behaviour of which depends on the value of $n$. As a general fact, we can establish the following

Proposition 3. Let $\mathfrak{n}_{n}^{k}$ be a nilpotent Lie algebra of the type in Equation (31) satisfying the quadratic Equation (35). Then, the following relations hold:

1. If $k \geq 6$ is even and $n \geq 4 k$, then $\mathfrak{n}_{n}^{k}$ is isomorphic to $Q_{n}$.

2. If $k \geq 7$ is odd and $n \geq 4 k$, then $\mathfrak{n}_{n}^{k}$ is either isomorphic to $Q_{n}$ or to the Lie algebra with brackets

$$
\begin{array}{ll}
{\left[X_{1}, X_{j}\right]=X_{j+1},} & 2 \leq j \leq n-2, \\
\left.X_{2}, X_{j}\right]=X_{j+k}, & 3 \leq j \leq n-1-k, \\
{\left[X_{i}, X_{n+1-i}\right]=(-1)^{i} X_{n},} & 2 \leq i \leq \frac{n}{2} .
\end{array}
$$

Clearly, the Jacobi conditions always admit the zero solution $\left(\alpha_{1}, \cdots, \alpha_{\ell}\right)=(0, \cdots, 0)$ with $\ell=\left[\frac{n-k-1}{2}\right]$ corresponding to a nilpotent Lie algebra isomorphic to $Q_{n}$. For any fixed dimension $n$, the number of Jacobi conditions is given by $\frac{1}{6} n(n-1)(n-2)$, while the number of parameters equals $\ell$. Now, while for the change of dimension $n \rightarrow n+2$ the number of parameters $\alpha_{i}$ increases by one unity, the number of new Jacobi conditions that appear is given by $n^{2}$, implying that the number of independent equations increases quadratically. Therefore, from a certain dimension onwards, the number of independent Jacobi conditions exceeds that of parameters, implying that only the trivial solution is possible. The only exception to this rule is given by the case of odd $k$ and $\left(\alpha_{1}, \cdots, \alpha_{\ell}\right)=$ $(1,0, \cdots, 0)$, where the coefficients $C_{2,3}^{k+3}=C_{2, q}^{q+k}$ for $4 \leq q \leq n-1-k$ always constitute a solution to the Jacobi conditions. The corresponding nilradical is the analog, contracting onto $Q_{n}$, of the nilradical $\mathfrak{f}_{n}$ of the so-called Bratzlavsky series contracting onto $L_{n}[12,36]$.

Technically, the systematized analysis of nilpotent Lie algebras admitting a one-dimensional maximal torus of derivations with eigenvalues $\operatorname{spec}(\mathfrak{t})=(1, k, k+1, \cdots, n+k-3, n+2 k-3)$ can be continued beyond $k=5$. However, even with the help of computer packages, we encounter dimensions where the solutions can only be obtained numerically, as the Jacobi conditions cannot be solved in closed form. The lowest value and dimension for which this occurs is given by $k=6$ and $d=22$, respectively. Supposed that $\mathfrak{n}_{22}^{6}$ is a Lie algebra of the type in Equation (31) and that $C_{2,3}^{9} \neq 0$ is satisfied, the solutions to the Jacobi conditions, besides two rational ones (see Table 1), correspond 
to solutions of the following system of linear and quadratic equations equations ( $\operatorname{As} \alpha_{1}=C_{2,3}^{9} \neq 0$, we can suppose that it equals 1):

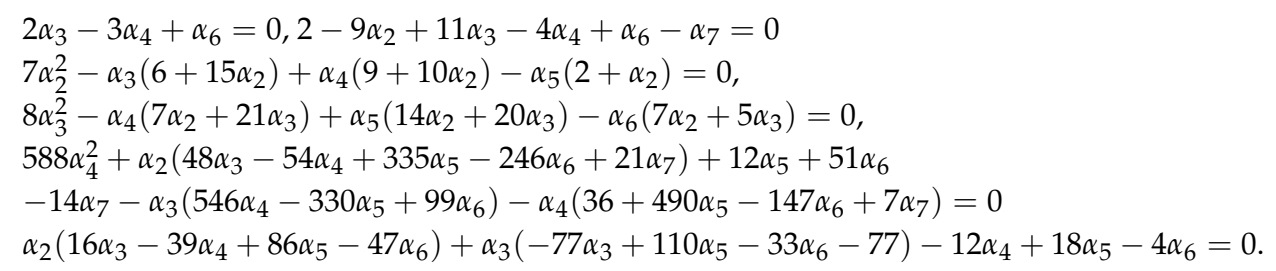

Table 1. Cohomologically rigid Lie algebras in dimensions $n \leq 30$ for $k \geq 6$.

\begin{tabular}{|c|c|c|c|}
\hline $\operatorname{dim} \mathfrak{n}$ & $k$ & $s$ & $\left(\alpha_{1}, \ldots, \alpha_{s}\right)$ \\
\hline 22 & 6 & 7 & $\left(0,0,0,1, \frac{74}{35}, 3,-1\right)$ \\
\hline 22 & 6 & 7 & $\left(0,1, \frac{3}{2}, \frac{9}{5}, \frac{5}{2}, \frac{15}{2}\right)$ \\
\hline 22 & 6 & 7 & $\left(1, \frac{(5 \pm \alpha)}{46}, \frac{(-21 \pm 5 \alpha)}{253}, \frac{(-21 \pm 5 \alpha)}{506}, 0, \frac{(21 \mp 5 \alpha)}{506}, \frac{(80 \mp 7 \alpha)}{253}\right), \alpha=\sqrt{301}$ \\
\hline 24 & 7 & 7 & $(0,0,0,0,1,-1,0)$ \\
\hline 24 & 7 & 7 & $\left(0,0,0,1, \frac{10}{7}, \frac{4}{7},-4\right)$ \\
\hline 26 & 7 & 8 & $\left(0,0,0,1,2, \frac{20}{7}, 4,7\right)$ \\
\hline 26 & 7 & 8 & $(0,0,0,0,0,1,-2,0)$ \\
\hline 26 & 7 & 8 & $\left(0,0,0,0,1, \frac{295}{126}, 3,-1\right)$ \\
\hline 26 & 7 & 8 & $\left(0,0,1, \frac{27}{22}, \frac{12}{11}, \frac{10}{11}, \frac{9}{11}, \frac{21}{22}\right)$ \\
\hline 26 & 7 & 8 & $\left(\frac{353 \pm 12 \alpha}{161}, 1, \frac{16 \mp 3 \alpha}{11}, \frac{138 \mp 30 \alpha}{121}, \frac{69 \pm 15 \alpha}{121}, 0, \frac{-69 \pm 15 \alpha}{121}, \frac{-169 \pm 42 \alpha}{121}\right), \alpha=\sqrt{26}$ \\
\hline 26 & 8 & 8 & $\left(1, \frac{25 \pm \alpha}{120}, \frac{15 \pm 3 \alpha}{520}, \frac{5 \pm \alpha}{120}, 0,0, \frac{5 \pm \alpha}{120}, \frac{-95 \pm 7 \alpha}{780}\right), \alpha=\sqrt{1585}$ \\
\hline 28 & 8 & 9 & $\left(0,0,1, \frac{61}{44}, \frac{31}{22}, \frac{15}{11}, \frac{16}{11}, \frac{91}{44}, 7\right)$ \\
\hline 28 & 8 & 9 & $(0,0,0,0,0,1,-1,0,0)$ \\
\hline 28 & 8 & 9 & $\left(0,0,0, \frac{42}{25}, \frac{64}{35}, \frac{268}{175}, \frac{7}{25},-\frac{308}{25}\right)$ \\
\hline 28 & 8 & 9 & $\left(0,0,0,0,1, \frac{265}{168}, \frac{71}{168},-4,1\right)$ \\
\hline 30 & 8 & 10 & $\left(0,0,0,0, \frac{17}{7}, \frac{1135}{294}, \frac{37}{7}, \frac{46}{7},-6\right)$ \\
\hline 30 & 8 & 10 & $\left(0,0,0,1, \frac{11}{20}, 1, \frac{5}{4}, \frac{10}{7}, \frac{7}{4}, \frac{14}{5}, \frac{21}{2}\right)$ \\
\hline 30 & 8 & 10 & $\left(0,0,0,0,0,1, \frac{387}{154}, 3,-1,0\right)$ \\
\hline 30 & 9 & 9 & $\left(0,0,1, \frac{23}{26}, \frac{5}{11}, \frac{25}{286},-\frac{25}{143},-\frac{119}{286},-\frac{126}{143}\right)$ \\
\hline 30 & 9 & 9 & $\left(0,0,0,0,1, \frac{20}{27},-\frac{40}{27},-\frac{115}{27}, 5\right)$ \\
\hline 30 & 9 & 9 & $(0,0,0,0,0,1,-2,1,0)$ \\
\hline 30 & 9 & 9 & $\left(0,0,0,1, \frac{64}{55}, \frac{5}{11},-\frac{10}{11},-\frac{37}{11},-\frac{112}{11}\right)$ \\
\hline 30 & 9 & 9 & $\begin{array}{c}\left(1, \frac{3(4720 \pm 27 \alpha}{28859}, \frac{3(196169 \pm 5665 \alpha)}{750334}, \frac{3(527147 \pm 16664 \alpha)}{9754342}, \frac{9(527147 \pm 16664 \alpha)}{9754342},\right. \\
\left.\quad 0,0, \frac{3(527147 \pm 16664 \alpha)}{9754342}, \frac{3(2194126 \pm 76151 \alpha}{9754342}\right), \alpha=\sqrt{1147}\end{array}$ \\
\hline
\end{tabular}


The solutions to these equations cannot be found in closed form, and require a numerical analysis. There are twelve solutions, divided into six real and six complex solutions. The real solutions (with a six digits approximation) are given by

$$
\begin{aligned}
& \left(\alpha_{2}, \cdots, \alpha_{7}\right)_{1}=(-0.672116,-0.689923,-2.3539,-3.77899,-5.68185,4.19364), \\
& \left(\alpha_{2}, \cdots, \alpha_{7}\right)_{2}=(-0.53422,0.401517,-0.507803,0.647213,-2.32645,10.9294), \\
& \left(\alpha_{2}, \cdots, \alpha_{7}\right)_{3}=(-0.268464,-0.425877,-0.212938,0,0.212938,0.796227), \\
& \left(\alpha_{2}, \cdots, \alpha_{7}\right)_{4}=(0.763277,0.27263,-0.00432786,-0.271782,-0.558244,-2.41149), \\
& \left(\alpha_{2}, \cdots, \alpha_{7}\right)_{5}=(0.485855,0.259869,0.129934,0,-0.129934,-0.163816), \\
& \left(\alpha_{2}, \cdots, \alpha_{7}\right)_{6}=(0.13456,-0.142263,-0.143553,-0.101978,-0.146134,-0.347857) .
\end{aligned}
$$

In these conditions, computing the cohomology with these values could lead to error due to the approximation of the values in the solution, hence an indirect approach for comparison is required. A quite laborious one consists in solving first the linear equations of the system and looking for a reduced set of polynomial equations equivalent to the system. Then, computing formally the cohomology with the diagonal entries $\left(1, \alpha_{2}, \cdots, \alpha_{7}\right)$ and introducing successively the constraints on the $\alpha_{i}$, a cumbersome computation allows us to establish that for the previous solutions the cohomology space $H^{2}\left(\mathfrak{r}_{22}^{6}, \mathfrak{r}_{22}^{6}\right)$ actually vanishes, so that these six nonrational solutions indeed provide cohomologically rigid Lie algebras (indeed, the direct computation with a nine digits approximation of the values $\alpha_{i}$ give a vanishing cohomology). As can be expected, the computing time for solving such cases for higher dimensions and values of $k$, even numerically, increases exponentially. This makes the separation of the solutions a difficult task, added to the fact that, for certain values of $k$ and $n$, several dozens of solutions may exist. Therefore, in the following, we focus only on the isolated solutions to the Jacobi conditions in Equation (35), especially emphasizing those solutions $\left(\alpha_{1}, \cdots, \alpha_{s}\right)$ that are rational.

\section{Cohomologically Rigid Algebras in Dimension $n \leq 30$}

In this section, we present the nilradicals of the type in Equation (31) that lead to real cohomologically rigid Lie algebras for values $k \geq 6$ and dimensions $n \leq 30$. For the reasons mentioned above, we restrict ourselves to the real solutions of Equation (35) that can be described in closed form, i.e., that do not require numerical analysis for their description. The nilradicals are given in terms of their fundamental parameters $\left(\alpha_{1}, \ldots, \alpha_{s}\right)$ (see Equation (36)) in tabular form, specifying the dimension of the nilradical $\mathfrak{n}$, the value of $k$ and the number of fundamental parameters.

Just as observed for the value $k=5$, for odd values of $k$ and dimensions $n \geq 30$, there begin to appear many solutions having non-vanishing cohomology that must be analyzed separately (unless they are the only nontrivial solution). The nonrational solutions also have a quite complicated form, thus, to find some pattern that leads to series of cohomologically rigid Lie algebras, it is in practice convenient to separate the rational solutions. Table 2 contains the rational solutions in dimensions $32 \leq n \leq 40$. 
Table 2. Rational cohomological rigid Lie algebras in dimensions $30<n \leq 40$.

\begin{tabular}{|c|c|c|c|}
\hline $\operatorname{dim} \mathfrak{n}$ & $k$ & $s$ & $\left(\alpha_{1}, \ldots, \alpha_{s}\right)$ \\
\hline 32 & 9 & 10 & $\left(0,0,0,0,0,1, \frac{56}{33}, \frac{10}{33},-4,1\right)$ \\
\hline 32 & 9 & 10 & $(0,0,0,0,0,0,1,-1,0,0)$ \\
\hline 32 & 9 & 10 & $\left(0,0,0,0,1,2, \frac{7}{3}, \frac{5}{3},-1,12\right)$ \\
\hline 32 & 9 & 10 & $\left(0,0,0,1, \frac{22}{13}, \frac{25}{13}, \frac{25}{13}, \frac{25}{13}, \frac{28}{13}, \frac{42}{13}\right)$ \\
\hline 32 & 10 & 10 & $\left(0,0,1,0,0, \frac{40}{37}, \frac{1359}{518}, \frac{120}{37}, \frac{34}{37},-36\right)$ \\
\hline 34 & 9 & 11 & $\left(0,0,0,0,0,1, \frac{14}{5}, \frac{791}{165}, \frac{32}{5}, \frac{31}{5},-6\right)$ \\
\hline 34 & 9 & 11 & $\left(0,0,0,0,1, \frac{30}{13}, \frac{45}{13}, \frac{175}{39}, \frac{75}{13}, \frac{108}{13}, \frac{210}{13}\right)$ \\
\hline 34 & 9 & 11 & $\left(0,0,0,1, \frac{8}{5}, \frac{22}{13}, \frac{20}{13}, \frac{35}{26}, \frac{16}{13}, \frac{84}{65}, \frac{24}{13}\right)$ \\
\hline 34 & 9 & 11 & $\left(0,0,0,0,0,0,1, \frac{413}{156}, 3,-1,0\right)$ \\
\hline 34 & 10 & 11 & $\left(0,0,0,0,1, \frac{10}{7}, \frac{41}{56},-\frac{82}{63},-\frac{283}{63},-\frac{62}{7}, \frac{130}{7}\right)$ \\
\hline 34 & 10 & 11 & $(0,0,0,0,0,0,1,-2,1,0,0)$ \\
\hline 34 & 10 & 11 & $\left(0,0,0,0,0,1, \frac{137}{65},-\frac{274}{65},-\frac{688}{165}, 5,-1\right)$ \\
\hline 34 & 10 & 11 & $\left(0,0,0,1, \frac{118}{91}, \frac{85}{91}, \frac{25}{91},-\frac{50}{91},-\frac{164}{91},-\frac{66}{13},-30\right)$ \\
\hline 34 & 11 & 10 & $\left(1,0,0,0, \frac{737}{988}, \frac{737}{546}, \frac{31691}{20748}, \frac{8107}{6916},-\frac{3685}{20748},-\frac{47168}{5187}\right)$ \\
\hline 36 & 10 & 12 & $(0,0,0,0,0,0,0,1,-1,0,0,0)$ \\
\hline 36 & 10 & 12 & $\left(0,0,0,0,1, \frac{188}{91}, \frac{243}{91}, \frac{109}{39}, \frac{695}{273}, \frac{162}{91},-\frac{114}{91},-\frac{462}{13}\right)$ \\
\hline 36 & 10 & 12 & $\left(0,0,0,0,0,0,1, \frac{70}{39}, \frac{8}{39},-4,1,0\right)$ \\
\hline 36 & 10 & 12 & $\left(0,0,0,0,0,1, \frac{151}{66}, \frac{3073}{1089}, \frac{1910}{1089},-\frac{71}{33},-\frac{773}{66}, 7\right)$ \\
\hline 36 & 10 & 12 & $\left(0,0,0,1, \frac{7}{4}, \frac{52}{26}, \frac{107}{52}, \frac{105}{52}, \frac{109}{52}, \frac{33}{13}, \frac{111}{26}, \frac{231}{13}\right)$ \\
\hline 36 & 12 & 11 & $\left(0,0,1,0,0, \frac{343}{933}, \frac{266}{933},-\frac{532}{933},-\frac{483}{311},-\frac{151}{933}, \frac{45374}{933}\right)$ \\
\hline 38 & 10 & 13 & $\left(0,0,0,0,1, \frac{13}{6}, 3, \frac{7}{2}, \frac{35}{9}, \frac{9}{2}, 6,11, \frac{99}{2}\right)$ \\
\hline 38 & 10 & 13 & $\left(0,0,0,0,0,1, \frac{135}{49}, \frac{33}{7}, \frac{1532}{231}, \frac{423}{9}, \frac{543}{49}, \frac{660}{49},-\frac{1188}{49}\right)$ \\
\hline 38 & 10 & 13 & $\left(0,0,0,0,0,0,0,1, \frac{124}{45}, 3,-1,0,0\right)$ \\
\hline 38 & 10 & 13 & $\left(0,0,0,0,0,0,1, \frac{378}{121}, \frac{8890}{1573}, \frac{892}{121}, \frac{711}{121},-6,1\right)$ \\
\hline 38 & 11 & 12 & $\left(0,0,0,0,1, \frac{23}{14}, \frac{10}{7}, \frac{1}{2},-1,-\frac{47}{14},-\frac{57}{7},-\frac{165}{7}\right)$ \\
\hline 38 & 11 & 12 & $\left(0,0,0,0,0,0,1, \frac{259}{286},-\frac{259}{143},-\frac{1171}{286}, 5,-1\right)$ \\
\hline 38 & 11 & 12 & $(0,0,0,0,0,0,0,1,-2,1,0,0)$ \\
\hline 38 & 11 & 12 & $\left(0,0,0,0,0,1, \frac{5}{3}, \frac{28}{33},-\frac{56}{33},-\frac{181}{33},-\frac{23}{3}, \frac{55}{3}\right)$ \\
\hline 38 & 11 & 12 & $\left(0,0,0,1, \frac{43}{34}, \frac{65}{68}, \frac{1}{2}, \frac{7}{68},-\frac{7}{34},-\frac{33}{68},-\frac{15}{17},-\frac{33}{17}\right)$ \\
\hline 40 & 11 & 13 & $(0,0,0,0,0,0,0,0,1,-1,0,0,0)$ \\
\hline 40 & 11 & 13 & $\left(0,0,0,0,1, \frac{35}{17}, \frac{91}{34}, \frac{49}{17}, \frac{49}{17}, \frac{49}{17}, \frac{105}{34}, \frac{66}{17}, \frac{231}{34}\right)$ \\
\hline 40 & 11 & 13 & $\left(0,0,0,0,0,0,0,1, \frac{516}{275}, \frac{34}{275},-4,1,0\right)$ \\
\hline 40 & 11 & 13 & $\left(0,0,0,0,0,0,1, \frac{28}{11}, \frac{5166}{1573}, \frac{2842}{1573},-\frac{35}{11},-\frac{126}{11}, 7\right)$ \\
\hline 40 & 11 & 13 & $\left(0,0,0,0,0,1, \frac{17}{7}, \frac{7}{2}, \frac{42}{11}, \frac{35}{11}, 1,-\frac{11}{2},-33\right)$ \\
\hline
\end{tabular}




\section{Some Series of Rigid Lie Algebras with $H^{2}\left(\mathfrak{r}_{n}^{k}, \mathfrak{r}_{n}^{k}\right)=0$}

As can be inferred from the tables, there are certain patterns in the (rational) solutions that suggest that the corresponding Lie algebras can be described for arbitrary values of $k$ (for even or odd values, respectively) while preserving the condition that the second cohomology space vanishes. The simplest of these series appears for even $k$, and has quite a low dimension, namely $k+6$, corresponding to the solution $\left(\alpha_{1}, \alpha_{2}\right)=(1,-2)$ of the Jacobi in Equation (35).

Proposition 4. Let $k \geq 2$ be an even integer and $\mathfrak{n}_{k+6}^{k}$ be the nilpotent Lie algebra with brackets

$$
\begin{array}{ll}
{\left[X_{1}, X_{j}\right]=X_{j+1},} & 2 \leq j \leq n-2 \\
{\left[X_{i}, X_{n+1-i}\right]=(-1)^{i} X_{n},} & 2 \leq i \leq \frac{n}{2} \\
{\left[X_{2}, X_{3}\right]=X_{j+k},} & j=3,4 \\
{\left[X_{2}, X_{5}\right]=3 X_{5+k},} & {\left[X_{3}, X_{4}\right]=-2 X_{5+k} .}
\end{array}
$$

Then, the solvable Lie algebra $\mathfrak{r}_{k+6}^{k}=\mathfrak{t} \vec{\oplus} \mathfrak{n}_{k+6}^{k}$ satisfies $H^{2}\left(\mathfrak{r}_{k+6}^{k}, \mathfrak{r}_{k+6}^{k}\right)=0$.

Besides this family, exclusive for even values $k$, there are other series that have very similar structure for both parities of $k$. The following result enumerates those series that begin for values of $k$ not exceeding twelve.

Proposition 5. The following Lie algebras $\mathfrak{n}_{n}^{k}$ of the type in Equation (31) satisfying the quadratic in Equation (35) lead to rank one cohomologically rigid Lie algebras $\mathfrak{r}_{n}^{k}$ with eigenvalue spectrum (28):

1. For odd $k \geq 7$ and dimension $n=4 k-4$ :

$$
\left(\alpha_{1}, \cdots, \alpha_{\frac{3 k-7}{2}}\right)=\left(0^{k-3}, 1,-1,0^{\frac{k-5}{2}}\right)
$$

2. For odd $k=2 q+1 \geq 7$ and dimension $n=4 k-2$ :

$$
\left(\alpha_{1}, \cdots, \alpha_{\frac{3 k-5}{2}}\right)=\left(0^{k-3}, 1, \varphi(q), 3,-1,0^{q-3}\right)
$$

where

$$
\varphi(q)=\frac{3(2 q-1)\left(10 q^{2}-11 q+2\right)}{2 q(2 q+1)(4 q-3)}, \quad q \geq 3 .
$$

3. For odd $k=2 q+1 \geq 9$ and dimension $n=4 k-4$ :

$$
\left(\alpha_{1}, \cdots, \alpha_{\frac{3 k-7}{2}}\right)=\left(0^{k-4}, 1, \varphi_{1}(q), \varphi_{2}(q),-4,1,0^{q-4}\right)
$$

where

$$
\varphi_{1}(q)=\frac{(q-1)(2 q-1)(11 q-12)}{q(2 q+1)(4 q-5)}, \varphi_{2}(q)=\frac{3\left(4-19 q+15 q^{2}-2 q^{3}\right)}{q(2 q+1)(4 q-5)}, \quad q \geq 3 .
$$

4. For odd $k=2 q \geq 11$ and dimension $n=4 k-6$ :

$$
\left(\alpha_{1}, \cdots, \alpha_{\frac{3 k-9}{2}}\right)=\left(0^{k-5}, 1, \phi_{1}(q), \phi_{2}(q), \phi_{3}(q), 5,-1,0^{q-5}\right)
$$


where

$$
\begin{array}{lc}
\phi_{1}(q)=\frac{(2 q-3)\left(20-37 q+14 q^{2}\right)}{2 q(2 q+1)(4 q-7)}, & \phi_{2}(q)=\frac{(3-2 q)\left(40-74 q+28 q^{2}\right)}{2 q(2 q+1)(4 q-7)}, \\
\phi_{3}(q)=\frac{\left(60-221 q+16 q^{2}+52 q^{3}\right)}{2 q(1+2 q)(7-4 q)}, & q \geq 5 .
\end{array}
$$

5. For even $k \geq 8$ and dimension $n=4 k-4$ :

$$
\left(\alpha_{1}, \cdots, \alpha_{\frac{3 k-6}{2}}\right)=\left(0^{k-3}, 1,-1,0^{\frac{k-4}{2}}\right)
$$

6. For even $k=2 q \geq 6$ and dimension $n=4 k-2$ :

$$
\left(\alpha_{1}, \cdots, \alpha_{\frac{3 k-4}{2}}\right)=\left(0^{k-3}, 1, \psi(q), 3,-1,0^{q-3}\right)
$$

where

$$
\psi(q)=\frac{3(q-1)\left(10 q^{2}-21 q+10\right)}{q(2 q-1)(4 q-3)}, \quad q \geq 3 .
$$

7. For even $k=2 q \geq 8$ and dimension $n=4 k-4$ :

$$
\left(\alpha_{1}, \cdots, \alpha_{\frac{3 k-6}{2}}\right)=\left(0^{k-4}, 1, \psi_{1}(q), \psi_{2}(q),-4,1,0^{q-4}\right)
$$

where

$$
\psi_{1}(q)=\frac{(q-1)(2 q-3)(22 q-35)}{2 q(2 q-1)(4 q-7)}, \psi_{2}(q)=\frac{3\left(35-71 q+36 q^{2}-4 q^{3}\right)}{2 q(2 q-11)(4 q-7)}, \quad q \geq 4 .
$$

8. For even $k=2 q \geq 10$ and dimension $n=4 k-6$ :

$$
\left(\alpha_{1}, \cdots, \alpha_{\frac{3 k-8}{2}}\right)=\left(0^{k-5}, 1, \psi_{1}(q), \psi_{2}(q), \psi_{3}(q), 5,-1,0^{q-5}\right)
$$

where

$$
\begin{array}{lc}
\psi_{1}(q)=\frac{(q-2)\left(42-51 q+14 q^{2}\right)}{q(2 q-1)(4 q-9)}, & \psi_{2}(q)=\frac{(2-q)\left(84-102 q+28 q^{2}\right)}{q(2 q-1)(4 q-9)} \\
\psi_{3}(q)=\frac{\left(84-99 q-31 q^{2}+26 q^{3}\right)}{q(1-2 q)(4 q-9)}, & q \geq 5 .
\end{array}
$$

The proof of the rigidity of these Lie algebras, as well as those of Proposition 5, is essentially the same, based on the Hochschild-Serre factorization theorem. In the following, we give an outline of the proof that is valid for all these cases. The first step consists in showing that for all these solvable algebras the derivations are all inner. To this extent, any 1-cochain $f \in C^{1}\left(\mathfrak{n}_{k}^{n}, \mathfrak{r}_{k}^{n}\right)$ can be written as

$$
f\left(X_{i}\right)=\sum_{l=1}^{n} a_{i}^{l} X_{l}+b_{i} T, 1 \leq i \leq n
$$

The invariance condition for cochains, as a consequence of the diagonal action of the torus generator $T$ on the nilradical, implies the following constraints

$$
f\left(X_{i}\right)=a_{i}^{i} X_{i}, 1 \leq i \leq n .
$$


If we now compute the coboundary operator for these cochains, we find that the only nonzero terms are the following:

$$
\begin{aligned}
d f\left(X_{1}, X_{j}\right) & =\left(a_{1}^{1}+a_{j}^{j}-a_{1+j}^{1+j}\right) X_{1+j}, 2 \leq j \leq n-2, \\
d f\left(X_{i}, X_{j}\right) & =\left(a_{i}^{i}+a_{j}^{j}-a_{i+j+k-2}^{i+j+k-2}\right) C_{i, j}^{i+j+k-2} X_{i+j+k-2}, 2 \leq i \leq k, i<j \leq n-i-1, \\
d f\left(X_{i}, X_{n-i+1}\right) & =\left(a_{i}^{i}+a_{n-i+1}^{n-i+1}-a_{n}^{n}\right) X_{n}, 2 \leq i \leq n-1 .
\end{aligned}
$$

Solving the resulting coefficient system allows us to find a basis of 1-cocycles $Z^{1}\left(\mathfrak{n}_{k}^{n}, \mathfrak{r}_{k}^{n}\right)^{\mathbf{t}}$, which can be chosen as

$$
f_{1}\left(X_{1}\right)=X_{1}, f_{1}\left(X_{i}\right)=(i+k-2) X_{i}, 2 \leq i \leq n-1, f_{1}\left(X_{n}\right)=(n+2 k-3) X_{n} .
$$

As the identity $\left[T, X_{\ell}\right]-f_{1}\left(X_{\ell}\right)=0$ holds for $1 \leq \ell \leq n$, we can identify the 1-cocycle with the adjoint operator ad $(T)$ of the torus generator, from which we conclude that $Z^{1}\left(\mathfrak{n}_{k}^{n}, \mathfrak{r}_{k}^{n}\right)^{\mathfrak{t}}=B^{1}\left(\mathfrak{n}_{k}^{n}, \mathfrak{r}_{k}^{n}\right)^{\mathfrak{t}}$, and further that $H^{1}\left(\mathfrak{n}_{k}^{n}, \mathfrak{r}_{k}^{n}\right)^{\mathfrak{t}}=H^{1}\left(\mathfrak{r}_{k}^{n}, \mathfrak{r}_{k}^{n}\right)=0$. The remaining cochains $f_{i}\left(X_{j}\right)=a_{i}^{j} X_{j}$ with $2 \leq i \leq n$ generate the space $B^{2}\left(\mathfrak{n}_{k}^{n}, \mathfrak{r}_{k}^{n}\right)^{\mathfrak{t}}$ and are actually linearly independent, thus $\operatorname{dim} B^{2}\left(\mathfrak{n}_{k}^{n}, \mathfrak{r}_{k}^{n}\right)^{\mathfrak{t}}=n-1$.

Consider now a generic 2-cochain that we can write as

$$
\varphi\left(X_{i}, X_{j}\right)=\sum_{k=1}^{n} \theta_{i, j}^{k} X_{k}+\eta_{i, j} T .
$$

If we evaluate the condition in Equation (15), we are led to the following constraints:

$$
\begin{aligned}
\varphi\left(X_{1}, X_{j}\right) & =\theta_{1, j}^{j+1} X_{j+1}, 2 \leq j \leq n-2 \\
\varphi\left(X_{i}, X_{j}\right) & =\theta_{i, j}^{i+j+k-2} X_{i+j+k-2}, 2 \leq i \leq j, i+j \leq n-k+1 \\
\varphi\left(X_{i}, X_{n-i+1}\right) & =\theta_{i, n-i+1}^{n} X_{n}, 2 \leq i \leq n-1 .
\end{aligned}
$$

Using the identities in Equations (32) and (33), the analysis of 2-cocycles reduces to evaluating the condition $d \varphi\left(X_{a}, X_{b}, X_{c}\right)=0$ for the triples $\left\{X_{1}, X_{i}, X_{j}\right\}$ and $\left\{X_{2}, X_{i}, X_{j}\right\}$. For the first triple, we get

$$
\begin{aligned}
d \varphi\left(X_{1}, X_{i}, X_{j}\right) & =\left(\theta_{i, j}^{i+j+k-2}-\theta_{i, j+1}^{i+j+k-1}-\theta_{i+1, j}^{i+j+k-1}\right) X_{i+j+k-1}, 2 \leq i+1<j, i+j \leq 2 k-3, \\
d \varphi\left(X_{1}, X_{i}, X_{n-i}\right) & =\left((-1)^{i} \theta_{1, i}^{i+1}+(-1)^{i+1} \theta_{1, n-i}^{n-i+1}-\theta_{i, n-i+1}^{n}-\theta_{i+1, n-i}^{n}\right) X_{n}, 2 \leq i \leq \frac{n}{2} \\
d \varphi\left(X_{1}, X_{i}, X_{j}\right) & =\left(-C_{i+1, j}^{i+j+k-1} \theta_{1, i}^{i+1}-C_{i, j+1}^{i+j+k-1} \theta_{i, j}^{i+j+k-1}+\theta_{i, j}^{i+j+k-2}-\theta_{i+1, j}^{i+j+k-1}-\theta_{i, j+1}^{i+j+k-1}\right) X_{i+j+k-1},
\end{aligned}
$$

where, in the last identity, $2 \leq i, i<j, 2 k-2 \leq i+j \leq n-k+1, i<k$.

For the triple $\left\{X_{2}, X_{i}, X_{j}\right\}$, the restrictions obtained are

$$
\begin{gathered}
d \varphi\left(X_{2}, X_{i}, X_{j}\right)=\left(C_{2, i}^{i+k} \theta_{j, i+k}^{i+j+2 k-2}-C_{2, j}^{j+k} \theta_{i, j+k}^{i+j+2 k-2}+C_{2, i+j+k-2} \theta_{i, j}^{i+j+k-2}+\right) X_{i+j+2 k-2}+ \\
+\left(C_{i, j}^{i+j+k-2} \theta_{2, i+j+k-2}^{i+j+2 k-2}-C_{i, j+k}^{i+j+2 k-2} \theta_{2, j+k}^{j+k}-C_{j, i+k}^{i+j+2 k-2} \theta_{2, i}^{i+k}\right) X_{i+j+2 k-2}
\end{gathered}
$$

for $3 \leq i, i<j, i+j \leq n-2 k+1, i<k$, as well as the equations

$$
\begin{aligned}
d \varphi\left(X_{2}, X_{i}, X_{n-k-i+1}\right) & =\left(\theta_{i, n-k-i+1}^{n-1}-(-1)^{i} \theta_{2, n-k-i+1}^{n-k-i+3}+(-1)^{i+k} \theta_{2, i}^{i+k}-C_{2, i}^{i+k} \theta_{i+k, n-k-i+1}^{n}\right. \\
& \left.-C_{2, n-k-i+1}^{n-i+1} \theta_{i, n-i+1}^{n}+C_{i, n-k-i+1}^{n-1} \theta_{2, n-1}^{n}\right) X_{n}, 3 \leq i \leq \frac{n}{2} .
\end{aligned}
$$


Now, let $\ell_{0}$ be the minimal index such that $\alpha_{\ell_{0}-1}=C_{\ell_{0}, \ell_{0}+1}^{k+2 \ell_{0}-1} \neq 0$. Rescaling, we can suppose that it has the value 1. The system of coefficients obtained from Equations (47)-(49) allows us, after some heavy algebraic manipulation, to obtain the solutions as

$$
\begin{aligned}
\theta_{i, j}^{i+j+k-2}= & C_{i, j}^{i+j+k-2}\left(-\sum_{t=2}^{i-1} \theta_{1, t}^{t+1}+\sum_{t=1}^{2 l+1-j} \theta_{1,2 l+1-t}^{2 l+2-t}-\sum_{t=1}^{j-2 l-1} \theta_{1,2 l+t}^{2 l+1+t}+\right. \\
& \left.\sum_{t=1}^{i+j-2 l-3} \theta_{1,2 l+t+k}^{2 l+t+k+1}+(-1)^{l+1} \theta_{2,2 l+1}^{2 l+k+1}\right), 2 \leq i<j, i+j \leq n-k+1 \\
\theta_{i, n-i+1}^{n}= & (-1)^{i}\left(-\sum_{t=1}^{i-2}\left(\theta_{1, t+1}^{t+2}+\theta_{1, n-1-t}^{n-t}\right)+\theta_{2, n-1}^{n}\right) X_{n}, 2 \leq i \leq \frac{n}{2} .
\end{aligned}
$$

Consequently, any 2-cocycle can be expressed as a linear combination of $\theta_{1, i}^{i+1}$ with $2 \leq i \leq n-2$, as well as $\theta_{2,2 l+1}^{2 k+k-1}$ and $\theta_{2, n-1}^{n}$. The corresponding cocycles are linearly independent, from which we conclude that $\operatorname{dim} Z^{2}\left(\mathfrak{n}_{k}^{n}, \mathfrak{r}_{k}^{n}\right)^{\mathfrak{t}}=n-1$, showing that $\operatorname{dim} H^{2}\left(\mathfrak{r}_{k}^{n}, \mathfrak{r}_{k}^{n}\right)=0$.

At this point, it is natural to ask whether the remaining Lie algebras covered in Tables 1 and 2 also belong to some family dependent on $k$. It is likely that many other series appear, although, as follows from the preceding analysis of the series in Proposition 5, the dimension of the first term in each family increases and dimensions of the nilradical beyond $n=100$ are required to recognize the coefficient pattern for the diagonal sequence in Equation (36). It is hoped to find a more systematic description in some future work.

\section{Conclusions}

We have studied some general properties of rank one solvable Lie algebras $\mathfrak{r}_{n}^{k}$ with an eigenvalue spectrum given by $\operatorname{spec}(\mathfrak{t})=(1, k, k+1, \cdots, n+k-3, n+2 k-3)$, corresponding to Lie algebras possessing a nilradical that contracts onto the model filiform algebra $Q_{n}$. This can be seen as a complementary study to that developed in [15], where rank one algebras the nilradical of which contracts onto $L_{n}$ are considered. We have particularly focused on those Lie algebras that have a vanishing Chevalley cohomology $\operatorname{dim} H^{2}\left(\mathfrak{r}_{k}^{n}, \mathfrak{r}_{k}^{n}\right)$. The detailed analysis for low values of $k$ allows us to extrapolate the results and obtain some series of cohomologically rigid algebras defined for any $k$ (within a parity).

An interesting observation emerges from the inspection of the rigid families enumerated in Proposition 5. The regularities observed there lead us to conjecture the existence, in the dimensions range $4 k-6 \leq n \leq 4 k-2$, of cohomologically rigid Lie algebras $\mathfrak{r}_{n}^{k}$, the nilradical of which is of the type in Equation (31) and satisfies Equation (35) for the values

$$
\left(\alpha_{1}, \cdots, \alpha_{M}\right)=\left(0^{k-\ell_{0}}, 1, \phi_{1}(q), \cdots, \phi_{\ell_{0}-2}(q),(-1)^{\ell_{0}-1} \ell_{0},(-1)^{\ell_{0}}, 0^{q-\ell_{0}}\right)
$$

where $M=\frac{3 k+1-2 \ell_{0}}{2}$ for $k=2 q+1, M=\frac{3 k+2-2 \ell_{0}}{2}$ for $k=2 q$, and $q \geq \ell_{0}$ and $\phi_{s}(q)$ for some combinatorial function of $q$. However, to confirm this hypothesis, further detailed analysis of the solutions of Equation (35) for higher values of $k$ and dimensions $n$ are necessary, in order to establish precisely what values of $\ell_{0}$ actually appear, the exact values for the coefficients $\phi_{s}(q)$ and whether the resulting solvable Lie algebras, if existing, have vanishing Chevalley cohomology for all admissible values of $k$. It may further be asked whether a global description of these Lie algebras is feasible by means of a generating function that covers simultaneously the case of even and odd values of $k$. The same question concerning a uniform description as families can be formulated for the irrational solutions, that also appear with certain regularities. Work in this direction is currently in progress. 
It shall be observed that, in addition to the cohomologically rigid solutions found, there are also geometrically rigid solvable rank one Lie algebras with eigenvalue spectrum $\operatorname{spec}(\mathfrak{t})=$ $(1, k, k+1, \cdots, n+k-3, n+2 k-3)$ and cohomology space $H^{2}(\mathfrak{r}, \mathfrak{r})$ of arbitrary dimension $d>1$, as can be expected from the analog analysis of rigid algebras with a nilradical of type $A_{n}^{k}$, where rigid examples with higher cohomology have been found [24]. However, for geometrically rigid Lie algebras, the cohomology is merely an accessory tool, and their detailed analysis requires other methods such as the Jacobi schemes $[8,43]$. The detailed analysis of rigid Lie algebras of this class with the eigenvalue spectrum considered in this work will be presented elsewhere.

Author Contributions: Conceptualization, R.C.-S. and F.O.G.; Methodology, R.C.-S.; Software, F.O.G.; Writing-Review \& Editing, R.C.-S.

Funding: The first author (RCS) was partially supported by the research project MTM2016-79422-P of the AEI/FEDER (EU).

Conflicts of Interest: The authors declare no conflict of interest.

\section{References}

1. Carles, R. Sur certaines classes d'orbites ouvertes dans les variétés d'algèbres de Lie. C. R. Acad. Sci. Paris 1981, $293,545-547$.

2. Segal, I.E. A class of operators which are determined by groups. Duke Math. J. 1951, 18, 221-265. [CrossRef]

3. Koszul, J.L. Homologie et cohomologie des algèbres de Lie. Bull. Soc. Math. Fr. 1950, 78, 65-127. [CrossRef]

4. Fialowski, A. Deformations and contractions of algebraic structures. Proc. Steklov Inst. Math. 2014, 286, 240-252. [CrossRef]

5. Gerstenhaber, M. On the deformations of rings and algebras. Ann. Math. 1964, 79, 59-103. [CrossRef]

6. Verona, A. Introducere in Coomologia Algebrelor Lie; Editura Academiei Rep. Soc.: Bucharest, Romania, 1974.

7. Zusmanovich, P. A converse to the second Whitehead Lemma. J. Lie Theory 2008, 18, 295-299.

8. Carles, R. Déformations dans les schémas définis par les identités de Jacobi. Ann. Math. Blaise Pascal 1996, 3, 33-62. [CrossRef]

9. Ancochea, J.M.; Goze, M. Le rang du système linéaire des racines d'une algèbre de Lie rigide résoluble complexe. Commun. Algebra 1992, 20, 875-887. [CrossRef]

10. Favre, G. Système des poids sur une algèbre de Lie nilpotente. Manuscr. Math. 1973, 9, 53-90. [CrossRef]

11. Ancochea, J.M.; Campoamor-Stursberg, R. Cohomologically rigid solvable real Lie algebras with a nilradical of arbitrary characteristic sequence. Linear Algebra Appl. 2016, 488, 135-147. [CrossRef]

12. Ancochea, J.M.; Campoamor-Stursberg, R. Rigidity-preserving and cohomology-decreasing extensions of solvable rigid Lie algebras. Linear Multilinear Algebra 2017, 66, 525-539. [CrossRef]

13. Carles, R. Sur certaines classes d'algèbres de Lie rigides. Math. Ann. 1985, 272, 477-488. [CrossRef]

14. Carles, R. Sur la cohomologie d'une nouvelle classe d'algèbres de Lie qui généralisent les sous-algèbres de Borel. J. Algebra 1993, 154, 310-334. [CrossRef]

15. Goze, M.; Ancochea, J.M. Algèbres de Lie rigides dont le nilradical est filiforme. C. R. Acad. Sci. Paris 1991, 312, 21-24.

16. Goze, M.; Ancochea, J.M. On the classification of rigid Lie algebras. J. Algebra 2001, 245, 68-91. [CrossRef]

17. Nijenhuis, A.; Richardson, R.W. Deformations of Lie algebra structures. J. Math. Mech. 1967, 17, 89-105. [CrossRef]

18. Page, S.S. A characterization of rigid algebras. J. Lond. Math. Soc. 1970, 2, 237-240. [CrossRef]

19. Rauch, G. Effacement et déformation. Ann. Inst. Fourier 1972, 22, 239-269. [CrossRef]

20. Richardson, R.W. On the rigidity of semi-direct products of Lie algebras. Pac. J. Math. 1967, 22, 339-344. [CrossRef]

21. Goze, M.; Ancochea, J.M. On the nonrationality of rigid Lie algebras. Proc. Am. Math. Soc. 1999, 127, 2611-2618.

22. Vergne, M. Cohomologie des algèbres de Lie nilpotentes. Application á l'étude de la variété des algébres de Lie nilpotentes. Bull. Soc. Math. France 1970, 98, 81-116. [CrossRef]

23. Ancochea, J.M.; Goze, M. Algorithme de construction des algèbres de Lie rigides. Publ. Math. Univ. Paris VII $1989,31,285-306$. 
24. Ancochea, J.M.; Campoamor-Stursberg, R.; Oviaño García, F. New examples of rank one solvable real rigid Lie algebras possessing a nonvanishing Chevalley cohomology. Appl. Math. Comput. 2018, 339, 431-440.

25. Mal'cev, A.I. Solvable Lie algebras. Izv. Akad. Nauk SSSR 1945, 9, 329-356.

26. Šnobl, L.; Winternitz, P. Classification and Identification of Lie Algebras; CRC Monograph Series; American Mathematical Society: Providence, RI, USA, 2014; Volume 33.

27. Carles, R. Sur la structure des algèbres de Lie rigides. Ann. Inst. Fourier 1984, 34, 65-82. [CrossRef]

28. Azcárraga, J.A.; Izquierdo, J.M. Lie Groups, Lie Algebras, Cohomology and Some Applications to Physics; Cambridge Univeisity Press: Cambridge, UK, 1995.

29. Campoamor-Stursberg, R. A comment concerning cohomology and invariants of Lie algebras with respect to contractions and deformations. Phys. Lett. A 2007, 362, 360-367. [CrossRef]

30. Fialowski, A.; de Montigny, M. On deformations and contractions of Lie algebras. J. Phys. A Math. Gen. 2005, 38, 6335-6349. [CrossRef]

31. Weimar-Woods, E. Contractions, generalized Inönü-Wigner contractions and deformations of finite-dimensional Lie algebras. Rev. Math. Phys. 2000, 12, 1505-1529. [CrossRef]

32. Tolpigo, A.K. On the cohomology of parabolic Lie algebras. Mat. Zamet. 1972 12, 251-255. [CrossRef]

33. Ancochea, J.M.; Campoamor-Stursberg, R.; Garcia Vergnolle, L.; Goze, M. Algèbres résolubles réelles algébriquement rigides. Monatsh. Math. 2007, 152, 187-195. [CrossRef]

34. Ancochea, J.M.; Campoamor-Stursberg, R.; Garcia Vergnolle, L. Les algèbres de Lie résolubles rigides réelles ne sont pas nécessairement complètement résolubles. Linear Algebra Appl. 2006, 418, 657-664. [CrossRef]

35. Ancochea, J.M.; Campoamor-Stursberg, R. Classification of solvable real rigid Lie algebras with a nilradical of dimension $n \leq 6$. Linear Algebra Appl. 2015, 451, 54-75. [CrossRef]

36. Bratzlavsky, F. Sur les algèbres admettant un tore d'automorphismes donné. J. Algebra 1974, 30, 305-316. [CrossRef]

37. Carles, R. Un exemple d'algèbres de Lie résolubles rigides, au deuxième groupe de cohomologie non nul et pour lesquelles l'application quadratique de D.S. Rim est injective. C. R. Acad. Sci. Paris 2985, 300, 467-469.

38. Rim, D.S. Deformation of transitive Lie algebras. Ann. Math. 1966, 83, 339-357. [CrossRef]

39. Dozias, J. Sur les dérivations des algèbres de Lie. C. R. Acad. Sci. Paris 1964, 259, 2748-2750.

40. Goze, M.; Hakimjanov, Yu. Sur les algèbres de Lie nilpotentes admettant un tore de dérivations. Manuscr. Math. 1994, 84, 115-124. [CrossRef]

41. Grozman, P.; Leites, D. SuperLie and Problems (to Be) Solved with it, Preprint MPI-2003-39, MPIM-Bonn. Available online: www.mpim-bonn.mpg.de (accessed on 15 November 2018).

42. Grozman, P.; Leites, D. MATHEMATICA aided study of Lie algebras and their cohomology. From supergravity to ballbearings and magnetic hydrodynamics. Trans. Eng. Sci. 1997, 15, 185-192.

43. Carles, R.; Márquez García, M.C. Different methods for the study of obstructions in the schemes of Jacobi. Ann. Inst. Fourier 2011, 61, 453-490. [CrossRef]

(C) 2019 by the authors. Licensee MDPI, Basel, Switzerland. This article is an open access article distributed under the terms and conditions of the Creative Commons Attribution (CC BY) license (http:/ / creativecommons.org/licenses/by/4.0/). 